\title{
Silver Nanoparticles Biosynthesized from Vaccinium myrtillus L. against Multiple Antibiotic Resistance and Biofilm Forming Escherichia coli and Pseudomonas aeruginosa
}

\author{
Nilay Ildiz ${ }^{1, *}$, Firdevs Korkmaz², Sadi Yusufbeyoğlu², Ayşe Baldemir Kilic ${ }^{3}$, İsmail Ocsoy ${ }^{4}$ \\ 'Department of Pharmaceutical Microbiology, Faculty of Pharmacy, Erciyes University,Kayseri, TURKEY. \\ ${ }^{2}$ Faculty of Pharmacy, Erciyes University, Kayseri, TURKEY. \\ ${ }^{3}$ Department of Pharmaceutical Botany, Faculty of Gulhane Pharmacy, University of Health Sciences, Ankara, TURKEY. \\ ${ }^{4}$ Department of Analytical Chemistry, Faculty of Pharmacy, Erciyes University, Kayseri, TURKEY.
}

\begin{abstract}
Background: Despite new innovations and process improvements, biofilm forming bacterial infections still pose a serious threat to patients. Silver nanoparticles (AgNPs) have been shown to have antibacterial properties and have been applied for surface manufacturing of many permanent medical devices at the same time. Therefore, we attempted to compare the performance of green synthesis of AgNPs and Vaccinium myrtillus L. plant extracts in terms of antibacterial and antibiofilm potential against multi drug resistant (MDR) biofilm forming Pseudomonas aeruginosa and Escherichia coli clinical strains. Materials and Methods: The biosynthesized AgNPs were characterized by UV-Visible spectroscopy. The antibacterial activity of the nanoparticles was determined by using disc diffusion and broth micro dilution method. Antibiofilm properties of nanoparticles have also been investigated by using scanning electron microscopy (SEM) and tissue culture plate (TCP) method. Results: Both extract and AgNP showed comparable bactericidal $(p<0,0001)$ and antibiofilm activity $(p<0,0001)$, but the mode of bacterial interaction and the degree of damage were completely different. Conclusion: For the first time with this study, extracts and also nanoparticles obtained from $V$. myrtillus were found to be effective in strains that have high biofilm activity and multiple drug resistance. Biosynthesized AgNPs were found to reduce planktonic cells as well as biofilm growth in a dose dependent manner. The results also supported the antibiofilm potential of AgNPs. This finding thus provides an idea of the development of silver nanoparticle-based biomaterials for use as effective surface modifying agents.
\end{abstract}

Key words: E. coli, P. aeruginosa, Biofilm, Vaccinium myritillus, Nanoparticle.

\section{INTRODUCTION}

The human health problems have increased in spite of major advances in science and technology today. The infectious diseases is one of the most important of these problems. Micro-organisms gain resistance to existing antimicrobial drugs, especially antibiotics. Management of acute bacterial skin and skin structure infections with a focus on patients at high risk of treatment failure. ${ }^{1}$ The most important reason for this is the wrong and unnecessary use of antibiotics. This is made comprehension of new drugs mandatory. Thus, the use of natural resources has come to the agenda again. ${ }^{2}$ Vaccinium myrtillus, which has been used as food for centuries, has gained medical importance and has been the subject of scientific studies. ${ }^{3}$ For infection to occur, the pathogenic micro-organism must first colonize the target surface. The colonization begins with the attachment of the microorganism to living and inanimate surfaces. Micro-organisms attaching to foreign bodies from inanimate surfaces form
Submission Date: 12-06-2020; Revision Date: 25-08-2020; Accepted Date: 23-11-2020

DOI: 10.5530/ijper.55.1s.53 Correspondence: Dr. Nilay Ildiz Department of Pharmaceutical Microbiology, Faculty of Pharmacy, Erciyes University, Kayseri, TURKEY. Phone no: +90-3522076666 Email id: nilaygucluer@ erciyes.edu.tr

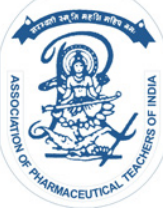

www.ijper.org 
biofilms. The role of biofilm in the pathogenesis of some chronic human infections is now widely accepted. The biofilm formation of Escherichia coli and Pseudomonas aeruginosa is responsible for serious infections in patients. ${ }^{4-6}$ Increased microbial resistance to traditional antibiotics has been hampered, as well as increased biofilm formation in some micro-organisms, highlighting the development of many antimicrobial NP, including AgNPs. In this study, the antimicrobial and antibiofilm effect on the biofilm-forming clinical strains was tested using $V$. myrtillus extract and silver nanoparticles (AgNPs) synthesized by extract using green synthesis.

\section{MATERIALS AND METHODS}

\section{Plant material and Preparation of anthocyanin rich extract}

Vaccinium fruits was collected from province Balikesir of Turkey in May 2016. The method of anthocyaninrich extract preparation was modified from Bagchi et al. (2004). The seeds of the fresh fruits were removed and cut into small pieces by passing through a shredder. The weighed 100 grams of fresh fruit was placed in a $500 \mathrm{~mL}$ beaker containing $100 \mathrm{ml}$ of distilled water. The mixture was microwaved $(900 \mathrm{~W})$ for 2 minutes. At the end of the process, Vaccinium extract was filtered and stored at $-20^{\circ} \mathrm{C}$ for further use.

\section{Green Synthesis and Characterization of Vaccinium myrtillus L. Silver Nanoparticles}

The extract is diluted to $50 \%$ and taken up in $2 \mathrm{~mL}$ of $50 \mathrm{mM} \mathrm{HCl}$ with $5 \mathrm{~mL}$ of $\mathrm{AgNO}_{3}$ nanoparticle was synthesized using the method of Zheng et al. (2015). ${ }^{8}$ Characterization of silver nanoparticles was carried out by UV-vis spectrophotometer.

\section{Bacterial strains}

Multi drug resistant strains of Escherichia coli and Pseudomonas aeruginosa were identified by the automated VITEK ${ }^{\circledR} 2$ system (bioMérieux) and obtained from Microbiology Laboratory, Necip Fazil City Hospital, Kahramanmaras. Total of 50 clinically resistant strains, 10 of them to have high biofilm activity with methods of qualitatively SEM and quantitatively by tissue culture plate methods. All methods were repeated three times and these 20 strains were included in the study.

\section{Antibacterial Activity of Extracts and Nanoparticles}

Antibacterial activities of all twenty strains were studied according to Clinical and Laboratory Standards Institute: According to CLSI standards using by Kirby Bauer disk diffusion method $^{9}$ measuring of inhibition zones and micro dilution method ${ }^{10}$ measuring of the minimum inhibitory concentration (MIC) values against extract and NPs.

\section{Detection of Biofilm}

Biofilm-forming strains were assessed by scanning electron microscopy (SEM, ZEISS LS-10 Life Science) and tissue culture plate methods. The isolated microorganism was inoculated in $10 \mathrm{ml}$ of trypticase soy broth (with $1 \%$ dextrose) and incubated at $37^{\circ} \mathrm{C}$ for $24 \mathrm{hr}$. The incubated broth was diluted 1:100 with fresh trypticase


culture was added to $10 \mathrm{ml}$ of fresh trypticase soy broth (with 1\% dextrose) using sterile micropipette tip and mixed well. Each of the three individual wells of sterile 96- well flat bottom polystyrene tissue culture treated plates was inoculated with $200 \mu \mathrm{l}$ of the diluted broth culture. For positive control, Staphylococcus aureus ATCC 25923 and Pseudomonas aeruginosa ATCC 27853 were used and they were incubated, diluted and added to tissue culture plates similar to the test isolate. For negative control, each of the three individual wells of the sterile 96-well flat bottom polystyrene tissue culture treated plates was inoculated with $200 \mu$ l of the uninoculated sterile trypticase soy broth with $1 \%$ dextrose. The tissue culture plates were incubated at $37^{\circ} \mathrm{C}$ for $24 \mathrm{hr}$. After incubation, the contents of each well were removed by gentle tapping and the wells were washed four times with $200 \mu \mathrm{l}$ of phosphate buffer saline to remove free floating bacteria. After washing, $200 \mu \mathrm{l}$ of $2 \%$ sodium acetate was added to each well and incubated for $15 \mathrm{~min}$ to fix the biofilm. After fixing, the plate was emptied and air-dried. $200 \mu \mathrm{l}$ of $0.1 \%$ crystal violet was added to each well and incubated for $15 \mathrm{~min}$ to stain the fixed biofilm. The excess stain was removed by washing with deionized water and the plate was air-dried. The optical densities (OD) of wells were obtained using ELISA reader (Ao Absorbance Micro plate Reader-Azure Bio systems) at a wavelength of $570 \mathrm{~nm}$. The mean OD value of negative control (ODnc) was calculated. The cutoff value was calculated from the ODnc, using the formula: $\mathrm{ODco}=\mathrm{ODnc}+3 \mathrm{x}$ standard deviation $(\mathrm{SD})$ of nc Average OD value of the test organism wells was calculated. Based on the OD value, the test organisms were classified according to Cristensen et al. (1985) ${ }^{11}$ as in mean $O D$ values $<0.120=$ None, $0.120-0.240=$ None $/$ weak and $\geq 0.240=$ High.

\section{Data analyses}

The extract and $\mathrm{Np}$ were analyzed three times and mean $\pm \mathrm{SD}$ was calculated. Graphs and statistical analysis 
were performed using GraphPad prism version 8.0.1 and Student $t$-test. $P$ value less than 0.05 was considered statistically significant.

\section{RESULTS AND DISCUSSION}

\section{Particle size and Characterization of Vaccinum myrtillus L. Silver Nanoparticles}

The particle size of the synthesized AgNPs was determined by SEM and characterized by UV-vis spectrophotometer. The formation of silver nanoparticles (AgNP) was confirmed by ultraviolet visible spectroscopy, where a surface-plasmon resonance absorption peak was observed between 420 and $430 \mathrm{~nm}$. The results are shown in Figure 1A and 1B.

\section{Visualization of biofilm by SEM}

The 400 mesh copper grids (Electronmicroscopy sciences, Hatfield, PA)were used for in situ visualization (Figure 2) of biofilm by SEM.

\section{Antimicrobial and AntiBiofilm Activity}

The extract and NPs were diluted at 1: 1, 1: 5, 1: 10, 1: 15, 1: 20, 1: 25,1: 30 ratio and antimicrobial activities were studied. Antimicrobial activity was not observed against $P$. aeruginosa strain 2,5,6,9 at concentration range. The antimicrobial activity of NPs was very effective than extract. Comparing the nanoparticle with the extract among the different MIC ratios, it is seen that the nanoparticles are effective at 1 to 2 fold less dilutions
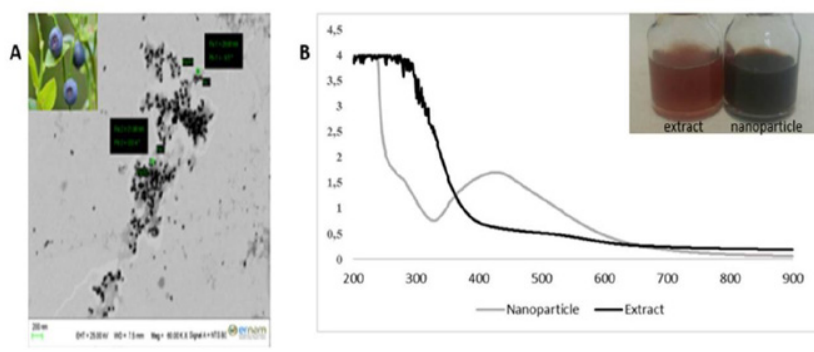

Figure 1: A) SEM image of nanoparticle and their size B) UV-vis spectrophotometer values.
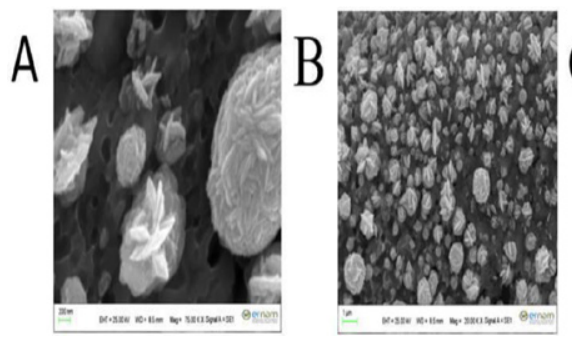

Figure 2: SEM image of biofilm formation A-B) $48 \mathrm{hr}$ C)72 hr incubation result.

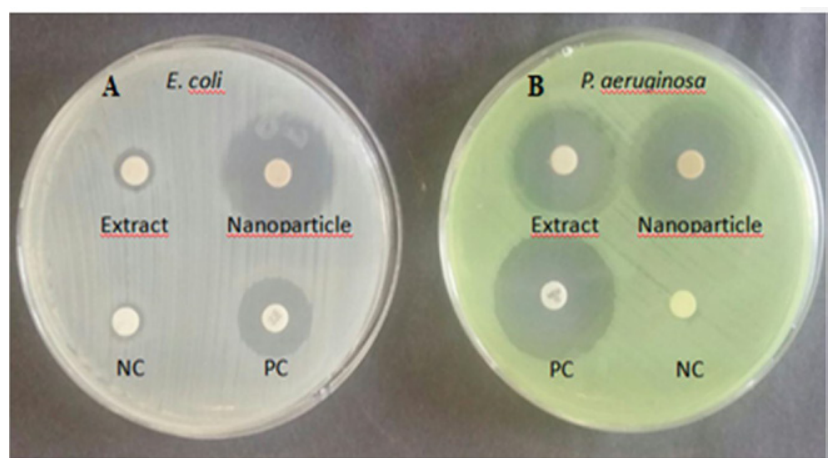

Figure 3: Disc diffusion results of A) E. coli B) $P$. aeruginosa.
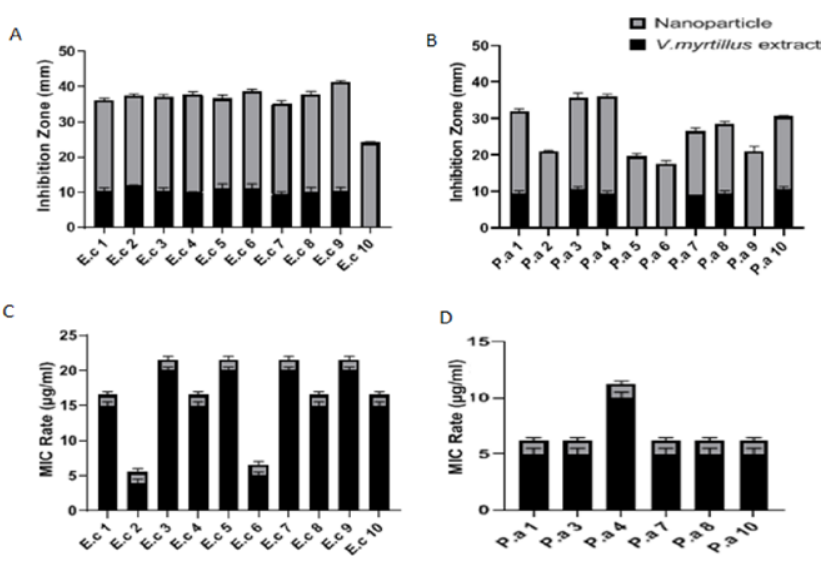

Figure 4: Antimicrobial activity results A-B) Disc Diffusion C-D) MICs.

*E.c=E.coli; P.a=P.aeruginosa

than the extract. All results were given Figure 3-4 and significant statistically $(\phi<0,0001)$.

The changes in the biofilm at the end of exposure $(2,8$, $18,24 \mathrm{hr}$ ) of the micro-organisms with extract and NPs were evaluated. The positive effect of samples on $E$. coli strains were observed at the end of $8 \mathrm{hr}$. But their effect on $P$. aeruginosa strains were effective after $18 \mathrm{hr}$ by only dissolving in biofilm. For the detection of changes in biofilms, tissue microplate method was evaluated by at 2, 8, 18, $24 \mathrm{hr}$ and compared with SEM images. As a result, the antibiofilm effect of the NP was about 15-fold higher than the extract for E. coli isolates and the biofilm formation was reduced higher than to nonto-weak range after $8 \mathrm{hr}(p<0,0001)$. The antibiofilm effect for $P$. aeruginosa isolates was about 5 times higher observed in NPs and its effect decreased from high to medium degree after $18 \mathrm{hr}(p<0,0001)$. The results of quantitative antibiofilm effect were similar to the qualitative SEM results as seen Figure 5.

As seen in Figure 3, it is seen that extracts and nanoparticles are more effective on E. coli biofilm than $P$. aeruginosa. Especially nanoparticles disrupt E. coli cell 
A

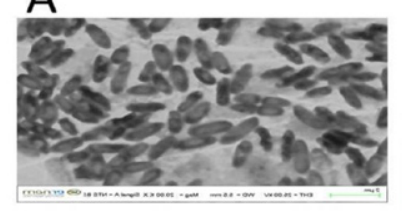

B
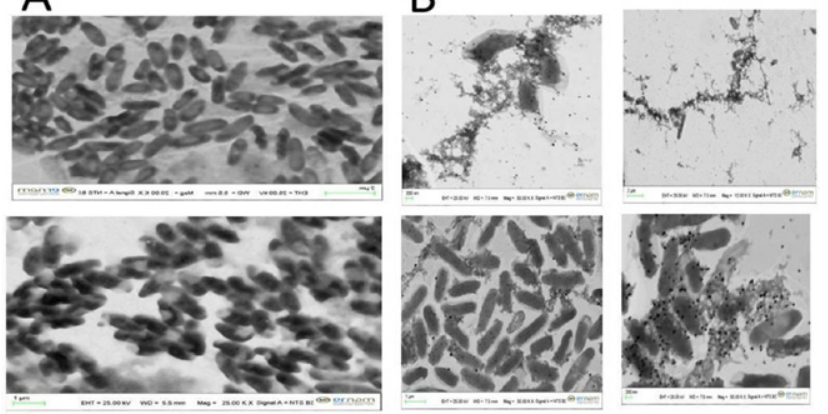

Figure 5: SEM images of Biofilm formation ( above -E. coli; below- $P$. aeruginosa).

A) Extracts B) NPs

wall, while on $P$. aeruginosa, the extract partially decreases the biofilm layer, however nanoparticles enter the bacteria.

Although there are some antimicrobial studies done with Vaccinium myrtillus, there is no contradiction regarding antibiofilm feature. For the first time with this study, extracts and also nanoparticles obtained from $V$. myrtillus were found to be effective in strains that have high biofilm activity and multiple drug resistance.

Benzoic acid is a simple aromatic carboxylic acid and is found naturally in many plant species. Many Vaccinium species, such as Vaccinium myrtillus, contain considerable amounts of benzoic acid in the fruits. ${ }^{12-16}$ The representative data obtained for benzoic acid derivatives such as vanillin, vanillic acid ${ }^{17}$ and gallic acid, ${ }^{18}$ has a biofilm inhibiting effect. This high antibiofilm effect may have been due to such compounds found intensely in this plant extract.

\section{CONCLUSION}

In this study, a simple and environmentally friendly method for AgNP synthesis is reported using V. myritillus extract. AgNPs were successfully synthesized from extract by green synthesis using silvernitrate solution. The effect of extract and AgNP on the biofilmforming clinical strains have beentested. Theresults of this study suggest that biofilm production should be investigated clinically in multi-drug resistant strains and that treatment of micro-organisms may be more easily achieved if biofilm inhibition is provided. Extract incorporated AgNPs are promising effect on biofilm formations.

\section{ACKNOWLEDGEMENT}

This work was supported by Tubitak-2209-A and Erciyes University TCD-2019-8016.

\section{CONFLICT OF INTEREST}

The authors declare no conflict of interest.

\section{ABBREVIATIONS}

AgNPs: Silver nanoparticles; MDR: Multi drug resistant; SEM: Scanning electron microscopy; TCP: tissue culture plate method; MIC: The minimum inhibitory concentration; OD: Optical density.

\section{REFERENCES}

1. Pulido-Cejudo A, Guzmán-Gutierrez M, Jalife-Montaño A, Ortiz-Covarrubias A, Martínez-Ordaz JL, Noyola-Villalobos HF, et al. Management of acute bacterial skin and skin structure infections with a focus on patients at high risk of treatment failure. Ther Adv Infect Dis. 2017;4(5):143-61.

2. Chouhan S, Sharma K, Guleria S. Antimicrobial Activity of Some Essential Oils-Present Status and Future Perspectives. Medicines. 2017;4(3):58.

3. Chu W, Cheung SCM, Lau RAW, Benzie IFF. Bilberry (Vaccinium myrtillus L.). Editors Source Herbal Medicine: Biomolecular and Clinical Aspects. $2^{\text {nd }}$ edition. Boca Raton (FL): CRC Press/Taylor and Francis. 2011;4.

4. Sanchez CJ, Mende K, Beckius ML, Akers KS, Romano DR, Wenke JC, et al. Biofilm formation by clinical isolates and the implications in chronic infections. BMC Infect Dis. 2013;13(1):47.

5. Piñero-Lambea C, Bodelón G, Fernández-Periáñez R, Cuesta AM, ÁlvarezVallina L, Fernández LA. Programming controlled adhesion of $E$. coli to target surfaces, cells and tumors with synthetic adhesins. ACS Synth Biol. 2015;4(4):463-73.

6. DiDomenico EG, Farulla I, Prignano G, Gallo MT, Vespaziani M, Cavallo I, et al. Biofilm is a major virulence determinant in bacterial colonization of chronic skin ulcers independently from the multidrug resistant phenotype. Int J Mol Sci. 2017;18(5):1077.

7. Bagchi D, Sen CK, Bagchi M, Atalay M. Anti-angiogenic, antioxidant and anticarcinogenic properties of a novel anthocyanin-rich berry extract formula. Biochem. 2004;69(1):75-80.

8. Zheng W, Fan H, Wang L, Jin Z. Oxidative self-polymerization of dopamine in an acidic environment. Langmuir. 2015;31(42):11671-7.

9. Clinical and Laboratory Standards Institute. Performance standards for antimicrobial disk susceptibility tests; approved standard M2-A10, $10^{\text {th }}$ ed. Clinical and Laboratory Standards Institute, Wayne, PA. 2009.

10. Clinical and Laboratory Standards Institute. Methods for dilution susceptibility tests for bacteria that grow aerobically; approved standard. M7-A8, $8^{\text {th }}$ ed. Clinical and Laboratory Standards Institute, Wayne, PA. 2009.

11. Christensen GD, Simpson WA, Younger JJ, Baddour LM, Barrett FF, Melton $\mathrm{DM}$,Beachey $\mathrm{EH}$. Adherence ofcoagulase-negative staphylococci to plastic tissue culture plates: Aquantitative model for the adherence of staphylococci to medical devices. J Clin Microbiol. 1985;22(6):996-1006.

12. Elkiran $\mathrm{O}$, Avşar $\mathrm{C}$. Chemical composition and biological activities of the essential oil from the leaves of Vaccinium myrtillus L. Bangladesh J Bot. 2020;49(1):91-6.

13. Ștefănescu BE, Călinoiu LF, Ranga F, Fetea F, Mocan A, Vodnar DC, et al. Chemical Composition and Biological Activities of the Nord-West Romanian Wild Bilberry (Vaccinium myrtillus L.) and Lingonberry (Vacciniumvitis-idaea L.) Leaves. Antioxidants. 2020;9(6):495.

14. Kowalska K, Olejnik A, Szwajgier D, Olkowicz M. Inhibitory activity of chokeberry, bilberry, raspberry and cranberry polyphenol-rich extract towards adipogenesis and oxidative stress in differentiated 3T3-L1 adipose cells. PloS one. 2017;12(11):1-15.

15. Jurikova T, Skrovankova S, Mlcek J, Balla S, Snopek L. Bioactive Compounds, Antioxidant Activity and Biological Effects of European Cranberry (Vaccinium oxycoccos). Molecules. 2018;24(1):24.

16. DelOlmo A, Calzada J, Nuñez M. Benzoic acid and its derivatives as naturally occurring compounds in foods and as additives: Uses, exposure and controversy. Crit Rev Food Sci Nutr. 2017;57(14):3084-103. 
17. Ponnusamy K, Paul D, Kweon JH. Inhibition of Quorum Sensing Mechanism and Aeromonashydrophila Biofilm Formation by Vanillin. Environ Eng Sci. 2009;26(8):1359-63.
18. Ta CAK, Arnason JT. Mini Review of Phytochemicals and Plant Taxa with Activity as Microbial Biofilm and Quorum Sensing Inhibitors. Molecules. 2016;21(1):29

\section{PICTORIAL ABSTRACT}



\section{SUMMARY}

- The antimicrobial and antibiofilm effect on the biofilm-forming clinical strains was tested using Vaccinium myritillus L. extract and silver nanoparticles (AgNPs) synthesized by extract using green synthesis.

- The plant extracts and AgNPs that were synthesized from extract found to be effective on multidrug resistant Gram-negative bacteria.

\section{About Authors}

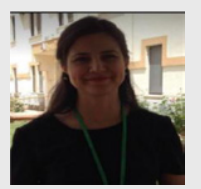

Dr. Nilay Ildiz, is the Head of Pharmaceutical Microbiology Dept in Erciyes University, Faculty of Pharmacy. She obtained her doctorate degree in Inönü University, Faculty of Medicine, Dept of Medical Microbiology in 2014. She continues to work on potential microbiological activities, Deveplopment of rapid disagnosis methods and recently on green chemistry.

Cite this article: Ildiz N, Korkmaz F, Yusufbeyoglu S, Kilic AB, Ocsoy I. Silver Nanoparticles Biosynthesized from Vaccinium myrtillus L against Multiple Antibiotic Resistance and Biofilm Forming Escherichia coli and Pseudomonas aeruginosa. Indian J of Pharmaceutical Education and Research. 2021;55(1s):s220-s224. 